\title{
On the Computation of Modified Bessel Function Ratios*
}

\author{
By Walter Gautschi and Josef Slavik
}

\begin{abstract}
A detailed comparison is made between a continued fraction of Gauss, and one of Perron, for the evaluation of ratios of modified Bessel functions $I_{\nu}(x) / I_{\nu-1}(x), x>0$, $\nu>0$. It will be shown that Perron's continued fraction has remarkable advantages over Gauss' continued fraction, particularly when $x>\nu$.
\end{abstract}

1. Introduction. Bessel functions are usually computed by a combination of several methods, including Taylor's series, recurrence relations, and asymptotic expansions. D. E. Amos et al. [1], [2] present a careful discussion of these various alternatives and implement them in the form of CDC 6600 computer subroutines. One of the building blocks of these subroutines is Miller's backward recurrence algorithm, which is used in combination with Taylor's series or asymptotic approximations to determine starting values for generating sequences of Bessel functions by downward recursion. As is well known [3], Miller's algorithm is equivalent to evaluating the continued fraction associated with the three-term recurrence relation for Bessel functions-a special case of Gauss' continued fraction. The purpose of this note is to draw attention to an alternative continued fraction, due to Perron, which can be used to compute ratios of modified Bessel functions, $I_{\nu}(x) / I_{\nu-1}(x), x>0, v>0$. Detailed comparisons with Gauss' continued fraction will show that Perron's continued fraction is substantially superior when $x \gg \nu$, and only moderately inferior otherwise. The advantage of Perron's continued fraction, unfortunately, is limited to modified Bessel functions of a real argument, for reasons indicated in [4].

2. The Continued Fractions of Gauss and Perron. From Gauss' continued fraction for ratios of hypergeometric functions (or, alternatively, from the recurrence relation satisfied by Bessel functions), one obtains the following continued fraction for ratios of modified Bessel functions [6, p. 349],

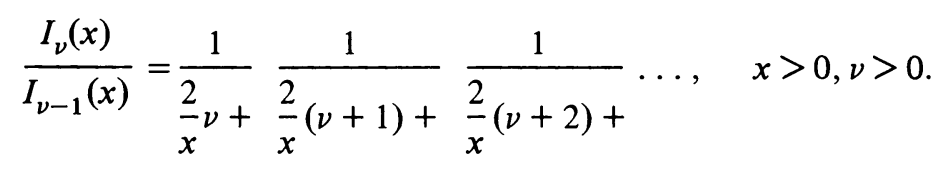

Equivalently,

$$
a_{0}(x) \frac{I_{\nu}(x)}{I_{\nu-1}(x)}=\frac{1}{1+} \frac{a_{1}(x)}{1+} \frac{a_{2}(x)}{1+} \ldots, \quad x>0, v>0
$$

Received June 20, 1977.

AMS (MOS) subject classifications (1970). Primary 33A40; Secondary 40A1 5, 65D15.

* The work of the first author was sponsored in part by the United States Army under Contract No. DAAG29-75-C-0024 while at the Mathematics Research Center, University of Wisconsin, Madison; and by the National Science Foundation under grant MCS 76-00842A01. 
where

$$
\left\{\begin{array}{l}
a_{0}(x)=\frac{2 v}{x}, \\
a_{k}(x)=\frac{\frac{1}{4} x^{2}}{(\nu+k-1)(\nu+k)}, \quad k=1,2,3, \ldots
\end{array}\right.
$$

We shall refer to (2.2), (2.1') briefly as the Gauss continued fraction. A less known continued fraction derives from Perron's continued fraction for ratios of confluent hypergeometric functions $[5$, p. 278],

$$
\frac{I_{\nu}(x)}{I_{\nu-1}(x)}=\frac{x}{2 v+x-} \frac{2 x\left(\nu+\frac{1}{2}\right)}{2 v+1+2 x-} \frac{2 x\left(\nu+\frac{3}{2}\right)}{2 v+2+2 x-} \frac{2 x\left(\nu+\frac{5}{2}\right)}{2 v+3+2 x-} \ldots
$$

or, equivalently, (2.2) with

$$
\left\{\begin{array}{l}
a_{0}(x)=\frac{x+2 v}{x}, \\
a_{1}(x)=-\frac{1}{2} x \frac{v+\frac{1}{2}}{\left(\nu+\frac{x}{2}\right)\left(\nu+x+\frac{1}{2}\right)}, \\
a_{k}(x)=-\frac{1}{2} x \frac{v+k-\frac{1}{2}}{\left(\nu+x+\frac{k-1}{2}\right)\left(\nu+x+\frac{k}{2}\right)}, k=2,3,4, \ldots
\end{array}\right.
$$

We refer to (2.2), $\left(2.3^{\prime}\right)$ briefly as the Perron continued fraction. The continued fraction in (2.2) can be written as an infinite series [6, p. 17ff.],

$$
\frac{1}{1+} \frac{a_{1}}{1+} \frac{a_{2}}{1+} \ldots=\sum_{k=0}^{\infty} p_{k}
$$

where

$$
p_{0}=1, \quad p_{k}=\rho_{1} \rho_{2} \ldots \rho_{k}, \quad k=1,2,3, \ldots
$$

and

$$
\rho_{0}=0, \quad \rho_{k}=\frac{-a_{k}\left(1+\rho_{k-1}\right)}{1+a_{k}\left(1+\rho_{k-1}\right)}, \quad k=1,2,3, \ldots,
$$

the $n$th partial sum of the series on the right of (2.4) being equal to the $n$th convergent of the continued fraction on the left, for $n=1,2,3, \ldots$.

In studying the convergence behavior of (2.4), it is convenient to introduce

$$
\sigma_{k}=1+\rho_{k}, \quad k=0,1,2, \ldots,
$$

and to rewrite (2.6) as 


$$
\sigma_{0}=1, \quad \sigma_{k}=\frac{1}{1+a_{k} \sigma_{k-1}}, \quad k=1,2,3, \ldots .
$$

Each $\sigma_{k}$ can be represented by a finite continued fraction,

$$
\sigma_{1}=\frac{1}{1+a_{1}}, \quad \sigma_{k}=\frac{1}{1+} \frac{a_{k}}{1+} \frac{a_{k-1}}{1+} \ldots \frac{a_{2}}{1+a_{1}}, \quad k=2,3,4, \ldots
$$

While both continued fractions (2.1) and (2.3) converge for all $x>0, v>0$, their precise convergence behavior is difficult to analyze. Qualitatively, however, we can make the following observations. Using superscripts $G$ and $P$ to distinguish between the Gauss and Perron continued fraction, one easily deduces from (2.7), (2.8), $\left(2.1^{\prime}\right)$ and $\left(2.3^{\prime}\right)$ that

$$
-1<\rho_{k}^{G}<0 \quad(k \geqslant 1), \quad \rho_{k}^{G} \sim-\frac{x^{2}}{4 k^{2}} \quad(\text { as } k \rightarrow \infty),
$$

and

$$
0<\rho_{k}^{P}<1 \quad(k \geqslant 1), \quad \rho_{k}^{P} \sim \frac{2 x}{k} \quad(\text { as } k \rightarrow \infty)
$$

Since the quantities $\left|\rho_{k}\right|$, according to (2.4) and (2.5), can be thought of as "instantaneous (geometric) convergence rates", we see from (2.10) that the Gauss continued fraction, at each instant (i.e., for each $k$ ), behaves like an alternating geometric series with ratio $\left|\rho_{k}^{G}\right|<1$, and ultimately converges superlinearly. A similar behavior is exhibited by the Perron continued fraction, whose terms $p_{k}$ are positive and monotonically decreasing, at instantaneous rates $\rho_{k}^{P}<1$. There is an important difference, however, in the asymptotic rate of convergence, inasmuch as $\rho_{k}^{G}=O\left(k^{-2}\right)$ and $\rho_{k}^{P}=$ $O\left(k^{-1}\right)$. Asymptotically, the Gauss continued fraction thus converges twice as fast as Perron's. Unless high accuracies are being considered, however, it is the initial convergence behavior (not the asymptotic one) that matters, and in this regard the Perron continued fraction has some remarkable advantages, particularly if $x \gg \nu$. This will be discussed empirically in Section 3, and analytically for large $\nu \gg k$ in Section 4.

\section{An Empirical Comparison Between Gauss' and Perron's Continued Fraction.} For initial orientation, we set up the lattice $x, v=1(1) 100$ in the $(x, v)$-plane, and at each point of the lattice determine the number of terms required in (2.4) to obtain a relative accuracy of $\epsilon=1 / 210^{-d}$. More specifically, for each of the two continued fractions we determine the smallest integer $k$ such that**

$$
\left|p_{k}\right| \leqslant \epsilon\left|\sum_{r=0}^{k} p_{r}\right|, \quad \text { where } \epsilon=\frac{1}{2} 10^{-d}
$$

** In a computer algorithm one might replace (3.1) by the simpler stopping criterion $\left|p_{k}\right| \leqslant \epsilon\left(1+p_{1}\right)$, which has the advantage of allowing the right-hand bound to be computed before the start of the iteration (2.5), (2.6), thus saving one multiplication in each iteration step. By (2.10) and (2.11) we have $0<1+p_{1}<\Sigma_{r=0}^{k} p_{r}$, so that the simplified criterion is in fact more stringent. 
If we denote the respective integers by $k_{\epsilon}^{G}$ and $k_{\epsilon}^{P}$, we can ask for the set of lattice points in which $k_{\epsilon}^{G} \leqslant k_{\epsilon}^{P}$. It turns out that these points lie on or above a line that has roughly slope 1 and is somewhat shifted to the right of the line $\nu=x$. Examples

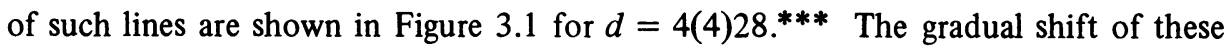
lines to the right, as $d$ increases, clearly expresses the superiority of Gauss' continued fraction for high accuracies, which is a result of its larger asymptotic rate of convergence (cf. Section 2).

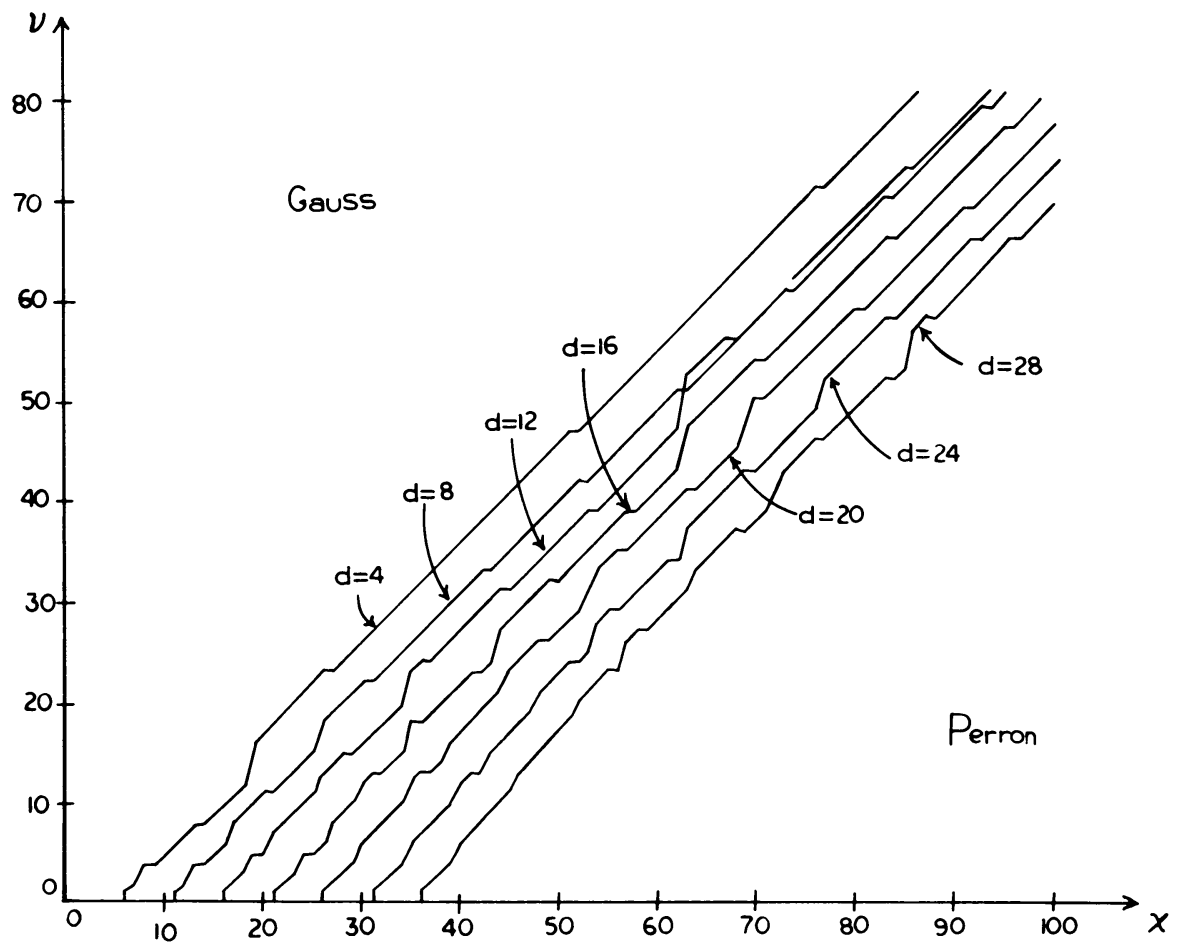

FIGURE 3.1. Lattice lines on and above of which $k_{\epsilon}^{G} \leqslant k_{\epsilon}^{P}$

If the better of the two continued fractions is used, the maximum number of iterations on the grid, $\mathrm{it}_{\mathrm{max}}=\max _{\mathrm{grid}} \min \left(k_{\epsilon}^{G}, k_{\epsilon}^{P}\right)$, is fairly small and increasing approximately linearly with $d$; see Table 3.1 .

\begin{tabular}{c|rrrrrrr}
$d$ & 4 & 8 & 12 & 16 & 20 & 24 & 28 \\
\hline $\mathrm{it}_{\max }$ & 8 & 15 & 22 & 29 & 36 & 43 & 51
\end{tabular}

TABLE 3.1. Maximum number of iterations

An indication of the relative merits of the two continued fractions can be had from Table 3.2, which shows $k_{\epsilon}^{G}$ and $k_{\epsilon}^{P}$ (under columns headed by " $G$ " and "P", respectively) in the case $d=8$ for selected values of $x$ and $\nu$. The striking superiority of the Perron continued fraction, when $x \gg \nu$, is particularly worth noting. Although

*** All computations were performed in single precision on the UNIVAC 1110 computer at the University of Wisconsin in Madison. 
the balance tips in favor of Gauss' continued fraction, when $x<<\nu$, the advantage is less significant there, since both continued fractions converge quite fast.

\begin{tabular}{|r|rr|rr|rr|rr|rr|rr|r|r|r|}
\hline \multirow{2}{*}{ X } & \multicolumn{2}{|c|}{10} & \multicolumn{2}{|c|}{20} & \multicolumn{2}{|c|}{40} & \multicolumn{2}{|c|}{60} & \multicolumn{2}{|c|}{80} & \multicolumn{2}{|c|}{100} \\
\cline { 2 - 14 } & G & P & G & P & G & P & G & P & G & P & G & P \\
\hline 10.0 & 9 & 13 & 7 & 11 & 5 & 9 & 4 & 8 & 4 & 7 & 4 & 7 \\
20.0 & 14 & 13 & 10 & 12 & 7 & 11 & 6 & 9 & 5 & 9 & 5 & 8 \\
40.0 & 21 & 10 & 16 & 12 & 11 & 12 & 8 & 11 & 7 & 11 & 6 & 11 \\
60.0 & 27 & 9 & 21 & 10 & 14 & 12 & 11 & 12 & 9 & 11 & 8 & 11 \\
80.0 & 32 & 8 & 26 & 10 & 18 & 11 & 13 & 12 & 11 & 12 & 9 & 11 \\
100.0 & 37 & 7 & 30 & 9 & 21 & 11 & 16 & 11 & 13 & 12 & 11 & 12 \\
\hline
\end{tabular}

\begin{tabular}{|r|rr|rr|}
\hline \multirow{2}{*}{$\mathbf{x}$} & \multicolumn{2}{|c|}{$V=1$} & \multicolumn{2}{|c|}{$V=\mathbf{x}$} \\
\cline { 2 - 5 } & $\mathrm{G}$ & $\mathrm{P}$ & $\mathrm{G}$ & $\mathrm{P}$ \\
\hline 100. & 45 & 5 & 11 & 12 \\
200. & 63 & 4 & 11 & 11 \\
500. & 100 & 4 & 11 & 11 \\
1000. & 142 & 3 & 11 & 11 \\
10000. & 452 & 3 & 12 & 11 \\
& & & & \\
\hline
\end{tabular}

TABLE 3.2. Number of iterations $k_{\epsilon}^{G}$ and $k_{\epsilon}^{P}$ for $d=8$

It should be observed, at this point, that the number of iterations in (2.5), (2.6) is not an entirely accurate measure of comparison between the two continued fractions, inasmuch as each iteration for $\rho_{k}^{G}$ requires slightly fewer arithmetic operations than each iteration for $\rho_{k}^{P}$. For Gauss' continued fraction, we have indeed

$$
\rho_{0}^{G}=0, \rho_{k}^{G}=\frac{-1 / 4 x^{2}\left(1+\rho_{k-1}^{G}\right)}{(\nu+k-1)(\nu+k)+1 / 4 x^{2}\left(1+\rho_{k-1}^{G}\right)}, \quad k=1,2,3, \ldots,
$$

while for Perron's continued fraction we have

$$
\left\{\begin{array}{l}
\rho_{0}^{P}=0, \quad \rho_{1}^{P}=\frac{1 / 2 x(\nu+1 / 2)}{\left(\nu+\frac{x}{2}\right)\left(\nu+x+\frac{1}{2}\right)-\frac{1}{2} x\left(\nu+\frac{1}{2}\right)}, \\
\rho_{k}^{P}=\frac{1 / 2 x(\nu+k-1 / 2)\left(1+\rho_{k-1}^{P}\right)}{\left(\nu+x+\frac{k-1}{2}\right)\left(\nu+x+\frac{k}{2}\right)-\frac{1}{2} x\left(\nu+k-\frac{1}{2}\right)\left(1+\rho_{k-1}^{P}\right)}, \\
k=2,3,4, \ldots .
\end{array}\right.
$$


The quantities $u_{k}=(\nu+k-1)(\nu+k)$ in (3.2) can be generated without multiplications as follows. Let $v_{k}=2(\nu+k)$; then $u_{0}=\nu(\nu-1), v_{0}=2 v$, and $u_{k}=u_{k-1}+$ $v_{k-1}, v_{k}=v_{k-1}+2$. (This is a refinement of a useful device employed by D. E. Amos et al. in [1, p. 84].) The iteration in (3.2) can thus be implemented in the form

$$
\left.\begin{array}{l}
\rho_{0}=0, \\
u_{0}=\nu(\nu-1), \quad v_{0}=2 \nu, \\
u_{k}=u_{k-1}+v_{k-1} \\
v_{k}=v_{k-1}+2 \\
t_{k}=x_{G}\left(1+\rho_{k-1}\right) \\
\rho_{k}=-t_{k} /\left(u_{k}+t_{k}\right)
\end{array}\right\}, \quad k=1,2,3, \ldots \quad\left(x_{G}=1 / 4 x^{2}\right),
$$

which requires four additions, one multiplication, and one division per iteration step. Similarly, we may implement (3.3) in the form

$$
\begin{aligned}
& \rho_{0}=0 \text {, } \\
& v_{0}=v+x_{P} \\
& v_{1}=\nu+x+1 / 2, \quad u_{1}=(\nu+x) v_{1}, \quad w_{1}=x_{P}(\nu+1 / 2), \\
& \rho_{1}=w_{1} /\left(v_{0} v_{1}-w_{1}\right), \\
& \left.\begin{array}{rl}
u_{k} & =u_{k-1}+v_{k-1} \\
v_{k} & =v_{k-1}+1 / 2 \\
w_{k} & =w_{k-1}+x_{P} \\
t_{k} & =w_{k}\left(1+\rho_{k-1}\right) \\
\rho_{k} & =t_{k} /\left(u_{k}-t_{k}\right)
\end{array}\right\}, \quad k=2,3,4, \ldots \quad\left(x_{P}=1 / 2 x\right),
\end{aligned}
$$

which requires five additions, one multiplication, and one division per iteration step. Thus, (3.3') involves one more addition than (3.2').

To complete a cycle in (2.4)-(2.6), one needs to compute

$$
p_{k}=\rho_{k} p_{k-1}, \quad s_{k}=s_{k-1}+p_{k},
$$

where $s_{k}=\Sigma_{r=0}^{k} p_{r}$, having initially set $p_{0}=s_{0}=1$. A complete cycle for the Gauss continued fraction thus costs five additions, two multiplications, and one division. For Perron's continued fraction, the count is the same, except for one more addition.

We take note of this slight disparity in work by replacing the lines in Figure 3.1 by straight lines of slope 1 passing through the points of intersection with the $x$-axis. In this way, Gauss' continued fraction is given a slightly more favorable treatment 
near the line of separation. From the data in Figure 3.1 one finds for the equation of these straight lines, empirically,

$$
\nu=\nu^{*}(x), \quad \nu^{*}(x)=x-1-1.25 d .
$$

We propose, therefore, to use Gauss' continued fraction whenever $\nu \geqslant \nu^{*}(x)$, and Perron's continued fraction otherwise.

Our confidence in this criterion is strengthened by testing the separation line (3.5) for other (in particular, larger) values of $x$, and for $\nu$ not necessarily integervalued. Given $\epsilon=1 / 210^{-d}$, we indeed compare $k_{\epsilon}^{G}$ with $k_{\epsilon}^{P}$ for selected values of $x$ and for $\nu$ in a small interval about $\nu=\nu^{*}(x)$. A typical result is shown in Table 3.3 for $d=12$, where $\nu_{j}=(1+j / 30) \nu^{*}(x), j=-3,-2, \ldots, 2,3$, and $x=50,100$, $200, \ldots, 6400$. Similar, equally satisfying, results are obtained for other values of d.

\begin{tabular}{|c|c|c|c|c|c|c|c|c|c|c|c|c|c|c|c|c|}
\hline & \multicolumn{2}{|c|}{$x=50$} & \multicolumn{2}{|c|}{$x=100$} & \multicolumn{2}{|c|}{$x=200$} & \multicolumn{2}{|c|}{$x=400$} & \multicolumn{2}{|c|}{$x=800$} & \multicolumn{2}{|c|}{$x=1600$} & \multicolumn{2}{|c|}{$x=3200$} & \multicolumn{2}{|c|}{$x=6400$} \\
\hline$v$ & G & $\mathrm{P}$ & G & $P$ & G & $P$ & G & $\mathbf{P}$ & G & $\mathbf{P}$ & G & $\mathbf{P}$ & G & $\mathbf{P}$ & G & $\mathbf{P}$ \\
\hline$v^{2}-3$ & 20 & 18 & 19 & 17 & 19 & 17 & 18 & 17 & 18 & 17 & 18 & 17 & 18 & 17 & 18 & 17 \\
\hline$v_{-2}$ & 20 & 18 & 19 & 17 & 18 & 17 & 18 & 17 & 18 & 17 & 18 & 17 & 18 & 17 & 18 & 17 \\
\hline$v_{-1}$ & 19 & 18 & 18 & 17 & 18 & 17 & 17 & 17 & 17 & 17 & 17 & 17 & 17 & 17 & 17 & 17 \\
\hline$\nu_{0}$ & 19 & 18 & 18 & 17 & 17 & 17 & 17 & 17 & 17 & 17 & 17 & 17 & 17 & 17 & 17 & 17 \\
\hline$v_{1}$ & 19 & 18 & 17 & 17 & 17 & 17 & 17 & 17 & 16 & 17 & 16 & 17 & 16 & 17 & 16 & 17 \\
\hline$v_{2}$ & 18 & 18 & 17 & 17 & 16 & 17 & 16 & 17 & 16 & 17 & 16 & 17 & 16 & 17 & 16 & 17 \\
\hline$v_{3}$ & 18 & 18 & 17 & 17 & 16 & 17 & 16 & 17 & 16 & 17 & 16 & 17 & 16 & 17 & 16 & 17 \\
\hline
\end{tabular}

TABLE 3.3. Comparison of $k_{\epsilon}^{G}$ and $k_{\epsilon}^{P}$ for $d=12$ near line of separation

With regard to computational work, it might seem more advantageous to use the simpler continued fractions in (2.1) and (2.3), and evaluate successive convergents "from tail to head". Thus, e.g., in case of the Gauss continued fraction,

$$
r_{n}^{(n)}=0, \quad r_{k}^{(n)}=\frac{1}{\frac{2}{x}(\nu+k)+r_{k+1}^{(n)}}, \quad k=n-1, n-2, \ldots, 0,
$$

where $r_{0}^{(n)}$ is the $n$th convergent of (2.1). Substituting again an addition for the multiplication in the denominator of (3.6), each iteration step (except for the first) requires two additions and one division. We must bear in mind, however, that the recursion (3.6) will have to be repeated at least once, possibly several times, with $n$ increased, in order to verify the accuracy attained. The amount of work then increases by a factor of two or more, making (3.6) about as expensive, if not more so, than $\left(3.2^{\prime}\right),(3.4)$. The only situation in which (3.6) might be preferable is when sharp and reliable estimates of $n$ are available for any given accuracy requirement, allowing one to take a single pass through the recursion (3.6). Similar remarks apply to Perron's continued fraction. 
The convergence character of both continued fractions is further revealed by examining the graphs of $\left|\rho_{k}^{G}\right|$ and $\left|\rho_{k}^{P}\right|$ as functions of $k$, for various values of $x$ and $\nu$. Increasing $\nu$ does not seem to alter much the character of these graphs, except for scaling them down in size. We therefore limit ourselves in displaying as typical the graphs for $\nu=1$. Those in Figure 3.2 are for the Gauss continued fraction, those in Figure 3.3 for Perron's continued fraction. It is seen, characteristically, that convergence of the Gauss continued fraction is hesitant, initially, and relatively slow, but eventually picks up speed and turns into rapid convergence. Perron's continued fraction, in contrast, starts out converging relatively fast, then slows down temporarily, before gradually regaining speed.

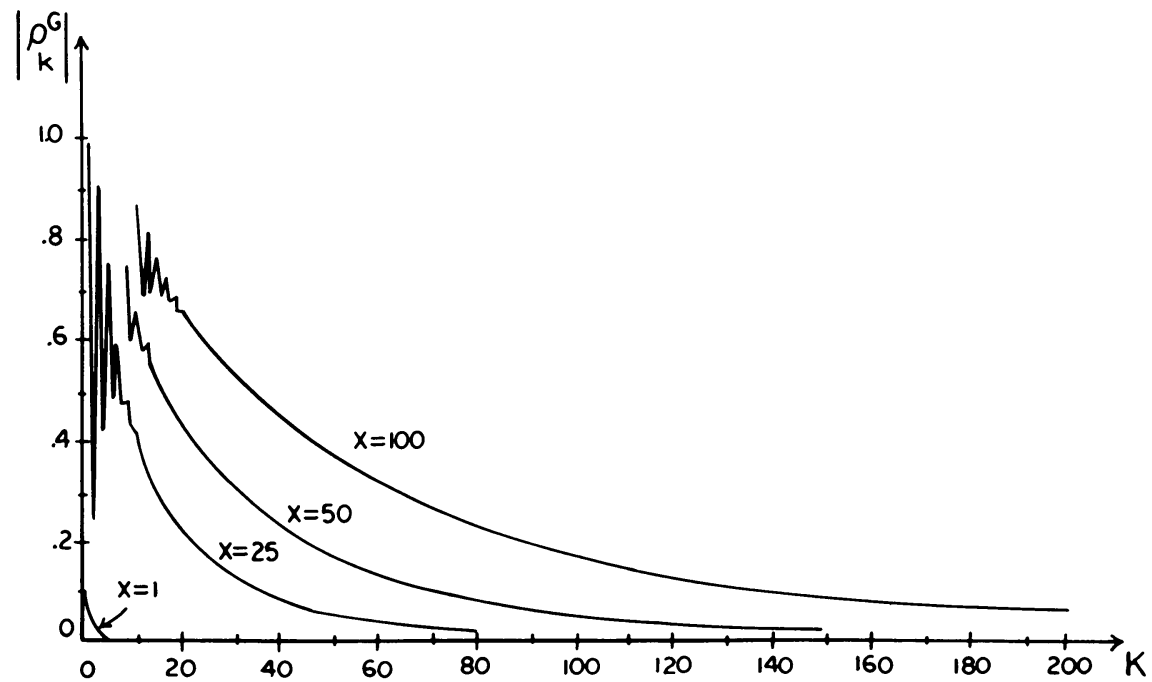

Figure 3.2. $\left|\rho_{k}^{G}\right|$ for $k=1(1) 2 \oint 0$ and $\nu=1, x=1,25,50,100$

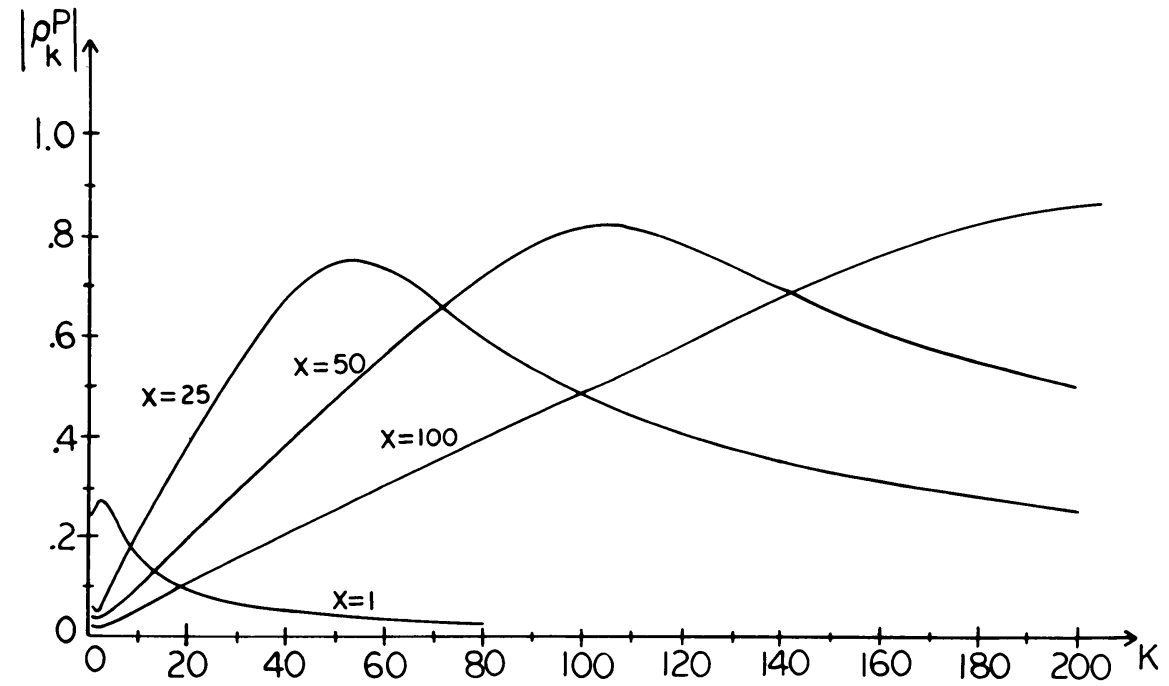

FIGURE 3.3. $\left|\rho_{k}^{P}\right|$ for $k=1(1) 200$ and $\nu=1, x=1,25,50,100$ 


\section{Initial Convergence Behavior of Gauss' and Perron's Continued Fraction for}

Large $\nu$. The case of large $\nu$ is of some interest in connection with the computation of starting values for subsequent generation of sequences of Bessel functions by downward recursion (cf. [1]). In this case, the initial convergence behavior of our continued fractions can be studied analytically, if certain simplifying approximations are made.

4.1. The Gauss Continued Fraction. If $\nu$ is large, and $k<<v$, the numerators $a_{k}$ in (2.2) are approximately constant,

$$
a_{k} \doteqdot a, \quad a=1 / 4 t^{2}, \quad k=1,2,3, \ldots,
$$

where, throughout this section,

$$
t=x / \nu \text {. }
$$

With this approximation, it then follows from (2.9) that

$$
\sigma_{k}=\frac{2}{1+s} \frac{1-\rho^{k+1}}{1-\rho^{k+2}}, \quad k \geqslant 0,
$$

where

$$
s=\sqrt{1+4 a}, \quad \rho=(1-s) /(1+s) .
$$

Clearly, $-1<\rho<0$, and therefore $0<\sigma_{k}<1$. Moreover,

$$
\left|\rho_{k}\right|=1-\sigma_{k}=|\rho| \frac{1-\rho^{k}}{1-\rho^{k+2}}, \quad k=0,1,2, \ldots
$$

An elementary computation will show $\left|\rho_{k+1}\right|<\left|\rho_{k}\right|$ and $\left|\rho_{k+2}\right|<\left|\rho_{k}\right|$, if $k$ is odd, and the reverse inequalities, if $k$ is even. The graph of $\left|\rho_{k}\right|$ on $k=1,2,3, \ldots$ therefore zig-zags towards a stationary level at $|\rho|$, whereby

$$
\left|\rho_{1}\right|>\left|\rho_{k}\right| \text { for all } k \geqslant 2 \text {. }
$$

(It should be noted that (4.4) does not represent correctly the behavior of $\left|\rho_{k}^{G}\right|$ as $k \rightarrow \infty$; cf. (2.10). This is because we assume here $k \ll<\nu$, where $\nu$ is large, but fixed.)

4.2. The Perron Continued Fraction. The approximations analogous to (4.1) are now

$$
a_{1} \doteqdot-\frac{1 / 2 t}{(1+1 / 2 t)(1+t)}
$$

$$
a_{k} \doteqdot a, \quad a=-\frac{\cdot 1 / 2 t}{(1+t)^{2}}, \quad k=2,3,4, \ldots,
$$

where $t$ again is given by (4.2). The continued fraction (2.9) then evaluates to

$$
\sigma_{k}=\frac{2}{1+s} \frac{1-\rho^{k}+\frac{2 a_{1}}{1+s}\left(1-\rho^{k-1}\right)}{1-\rho^{k+1}+\frac{2 a_{1}}{1+s}\left(1-\rho^{k}\right)}, \quad k \geqslant 1
$$


where $s$ and $\rho$ are as before in (4.3). Since $-1 / 8 \leqslant a<0$, we now have $0<\rho<$ $(\sqrt{2}-1) /(\sqrt{2}+1)=.17157 \ldots$ Noting that $1-\rho^{k+1}+\left(2 a_{1} /(1+s)\right)\left(1-\rho^{k}\right)>$ $\left(1-\rho^{k}\right)\left(1+2 a_{1} /(1+s)\right) \geqslant 0$ for $k \geqslant 0$, we conclude $\sigma_{k}>0$ for all $k \geqslant 1$, and, after a little computation, that $\sigma_{k}$ is strictly decreasing. Therefore,

$$
1<\frac{2}{1+s}<\sigma_{k} \leqslant \frac{1}{1+a_{1}} \leqslant \frac{1+\sqrt{2}}{2}=1.2071 \ldots,
$$

and it follows that

$$
\rho_{k}=\sigma_{k}-1=\rho \frac{1-\rho^{k-1}+\frac{2 a_{1}}{1+s}\left(1-\rho^{k-2}\right)}{1-\rho^{k+1}+\frac{2 a_{1}}{1+s}\left(1-\rho^{k}\right)}, \quad k=1,2,3, \ldots
$$

Since $\sigma_{k}$ decreases, we have

$$
0<\rho_{k+1}<\rho_{k} \text { for } k=1,2,3, \ldots,
$$

from which again follows the validity of (4.5).

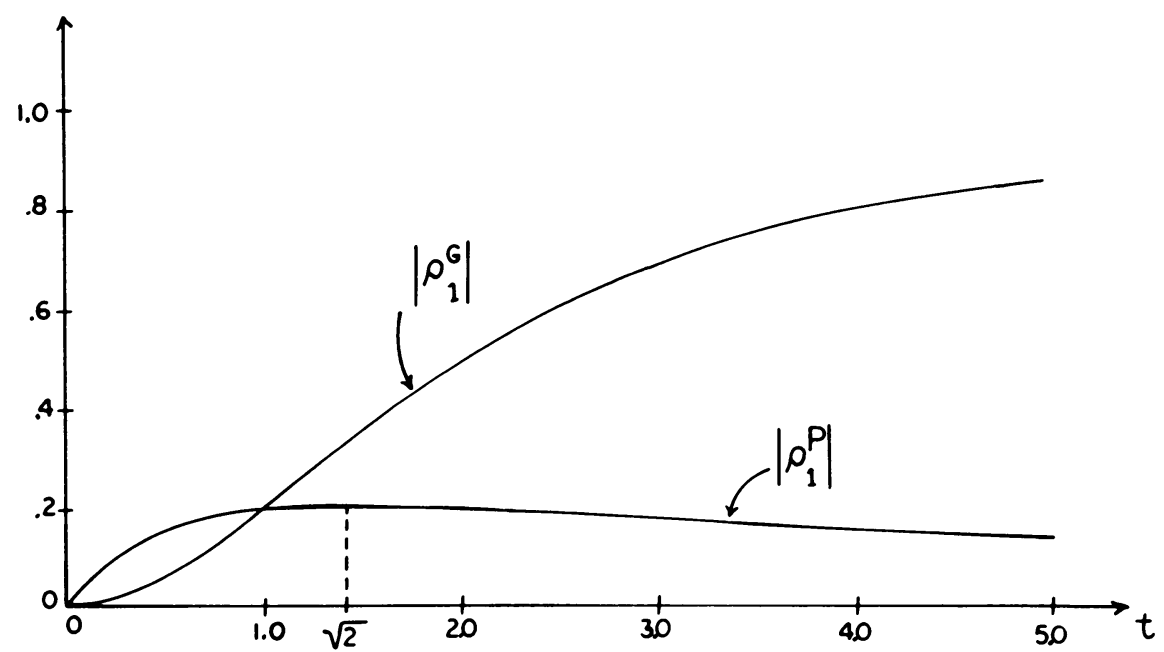

FIGURE 4.1. $\left|\rho_{1}^{G}\right|$ and $\left|\rho_{1}^{P}\right|$ as functions of $t=x / \nu$

4.3. Choosing Between Gauss' and Perron's Continued Fraction. Inequality (4.5) suggests to give preference to Gauss' continued fraction whenever

$$
\left|\rho_{1}^{G}\right| \leqslant\left|\rho_{1}^{P}\right|
$$

In view of

$$
\left|\rho_{1}^{G}\right|=\frac{t^{2}}{4+t^{2}}, \quad\left|\rho_{1}^{P}\right|=\frac{t}{2+2 t+t^{2}}, \quad t=\frac{x}{v},
$$

we have (4.8) if and only if $t^{3}+t^{2}+2 t-4=(t-1)\left(t^{2}+2 t+4\right) \leqslant 0$, i.e., if and only if 
With this choice we find that (cf. Figure 4.1)

$$
\left|\rho_{k}\right|<\left|\rho_{1}\right| \leqslant 1 / 2(\sqrt{2}-1)=.2071 \ldots, \quad \text { all } k \geqslant 2 \text {, }
$$

for both Gauss' and Perron's continued fraction (when selected according as (4.9) does or does not hold). Our choice, therefore, guarantees an initial rate of convergence of at least about .2 .

The criterion (4.9), having been derived by minimizing the "initial rate of convergence", naturally cannot be expected to optimize with regard to overall computational effort. Nevertheless, it is seen that (4.9) is in essential agreement with the empirical criterion $\nu \geqslant \nu^{*}(x)$ of (3.5), which indeed takes into account the overall computational work involved.

Department of Computer Sciences

Purdue University

Lafayette, Indiana 47907

Institut für Physikalische Weltraumforschung

Heidenhofstrasse 8

78 Freiburg i. Br., West Germany

1. D. E. AMOS, S. L. DANIEL \& M. K. WESTON, "CDC 6600 subroutines IBESS and JBESS for Bessel functions $I_{\nu}(x)$ and $J_{\nu}(x), x \geqslant 0, \nu \geqslant 0$," ACM Trans. Math. Software, v. 3, 1977, pp. 76-92.

2. D. E. AMOS, S. L. DANIEL \& M. K. WESTON, "Algorithm 511-CDC 6600 subroutines IBESS and JBESS for Bessel functions $I_{\nu}(x)$ and $J_{\nu}(x), x \geqslant 0, v \geqslant 0, " A C M$ Trans. Math. Software, v. 3, 1977, pp. 93-95.

3. W. GAUTSCHI, "Computational aspects of three-term recurrence relations," SIAM Rev., v. 9, 1967, pp. 24-82.

4. W. GAUTSCHI, "Anomalous convergence of a continued fraction for ratios of Kummer functions," Math. Comp., v. 31, 1977, pp. 994-999.

5. O. PERRON, Die Lehre von den Kettenbrüchen, vol. II, 3rd ed., Teubner Verlag, Stuttgart, 1957.

6. H. S. WALL, Analytic. Theory of Continued Fractions, Van Nostrand, New York, 1948. 